\title{
FOURIER SERIES ON COMPACT LIE GROUPS
}

MICHAEL E. TAYLOR

In [1], Gong Shang proves that the Fourier series of a sufficiently smooth function on a unitary group $U(n)$ converges absolutely and uniformly. Using the theory of elliptic operators, we give a short proof of a more general assertion.

Suppose $m$ is a finite strictly positive measure on a compact $C^{\infty}$ manifold $M$. Suppose $L$ is a strictly elliptic operator of order $k$ on $M$ which is selfadjoint on the space $L^{2}(M)$ of square in tegrable functions with respect to $m$. Then it is well known that for a sufficiently large positive scalar $\lambda,(\lambda+L)^{-1}$ is defined and compact, so $L$ has a complete set of eigenfunctions $\phi_{i}$ in $L^{2}(M)$, and any function $f \in L^{2}(M)$ can be expanded in a Fourier series $f=\sum \alpha_{i} \phi_{i}$ with respect to these eigenvectors, the series converging in Hilbert space norm. We ask the following question: what smoothness conditions can we place on $f$ to guarantee that the sum $f=\sum\left(f, \phi_{i}\right) \phi_{i}$ converges absolutely and uniformly? The following theorem appears to be well known, but we shall record the proof, which is quite simple. In what follows, $H_{k s}$ will be the Sobolev space of functions whose weak derivatives of order $\leqq k s$ are square integrable.

THEOREM 1. With notations as above, let $n$ be the dimension of $M$, and let $s \geqq n / 2 k$ be an integer. Suppose $f \in H_{k s}$. Then the Fourier series of . with respect to the eigenvectors of $L$ converges absolutely and uniformlyf

Proof. We use the fundamental fact that for a sufficiently large positive scalar $\lambda,(\lambda+L)^{s}: H_{k s} \rightarrow L^{2}(M)$ is a bicontinuous linear bijection. If $g=(\lambda+L)^{\circ} f$, we write the $L^{2}$-norm convergent series of $g, g=\sum_{i=1}^{\infty} \beta_{i} \phi_{i}$. Since $\left\|\sum_{i=n}^{\infty} \beta_{i} \phi_{i}\right\| \rightarrow 0$ as $n \rightarrow \infty$ it follows from the fact that $(\lambda+L)^{-s}$ is continuous that

$$
\left\|\sum_{i=n}^{\infty} \alpha_{i} \phi_{i}\right\|_{k s}=\left\|(\lambda+L)^{-s}\left(\sum_{i=n}^{\infty} \beta_{i} \phi_{i}\right)\right\|_{k s} \rightarrow 0
$$

as $n \rightarrow \infty$, where \|\| is the $L^{2}$ norm and \|\|$_{k s}$ is the Sobolev space norm. By Sobolev's inequality it follows that $\sup _{x \in M}\left|\sum_{i=n}^{\infty} \alpha_{i} \phi_{i}(x)\right|$ $\rightarrow 0$ as $n \rightarrow \infty$, so in fact $\sum_{i=1}^{\infty} \alpha_{i} \phi_{i}$ does converge uniformly to $f$. Finally, for a given $x_{0} \in M$, if we pick complex numbers $\omega_{i}$ of absolute value 1 such that $\omega_{i} \alpha_{i} \phi_{i}\left(x_{0}\right) \geqq 0$ for each $i$, then the same reasoning as above gives $\sup _{x \in M}\left|\sum_{i=n}^{\infty} \omega_{i} \alpha_{i} \phi_{i}(x)\right| \rightarrow 0$, and a fortiori

Received by the editors May 9, 1967. 


$$
\sum_{i=n}^{\infty}\left|\alpha_{i} \phi_{i}\left(x_{0}\right)\right| \rightarrow 0 \text { as } n \rightarrow \infty,
$$

which takes care of absolute convergence.

Now suppose $G$ is a compact Lie group. Let $L$ be the LaplaceBeltrami operator on $G$, endowed with a bi-invariant Riemannian metric. Let $m$ be its Haar measure. Then $L$ is a selfadjoint elliptic operator on $L^{2}(G)$. (Various implications of the idea of considering invariant Laplacians on Lie groups are discussed in [3].) We pick a complete set of inequivalent irreducible unitary representations $\left\{\pi_{k}\right\}$ of $G$. We can write $\pi_{k}$ as a matrix $\left(g_{i j}^{k}\right)$, and letting $n_{k}$ be the dimension of the representation space of $\pi_{k}$, we have that the $n_{\mathbf{k}}^{1 / 2} g_{i j}^{\boldsymbol{k}}(x)$ form an orthonormal basis of $L^{2}(G)$, by the orthogonality relations for compact groups and the Peter-Weyl theorem. By the Fourier series of a function $f \in L^{2}(G)$ is meant the Fourier series with respect to this orthonormal basis. Now $L$ belongs to the center of both the right and left invariant universal algebras (see Helgason [2, Chapter $\mathrm{X}$, Lemma 2.3]), and hence $L$ commutes with the representation $V$ of $G \times G$ on $L^{2}(G)$ defined by $V(x, y) f(z)=f\left(x^{-1} z y\right)$. We apply this observation after proving the following lemma.

LemMa. Let $\pi$ be an irreducible unitary representation of the compact group $G$, with matrix $\pi(x)=\left(g_{i j}(x)\right)$. Then the representation $T$ of $G \times G$ on the vector space spanned by the entry functions $g_{i j}(x)$, defined by $T(x, y) g_{i j}(z)=g_{i j}\left(x^{-1} z y\right)$, is irreducible.

Proof. Let $n$ be the degree of $\pi$, so the degree of $T$ is $n^{2}$. Now $T$ is irreducible if and only if $\int_{G \times G}|\operatorname{tr} T(x, y)|^{2} d x d y=1 / n^{2}$. But since $T(x, y) g_{i j}(z)=\sum g_{i k}\left(x^{-1}\right) g_{k l}(z) g_{l j}(y)$, we see that

$$
\operatorname{tr} T(x, y)=\sum_{i, j} g_{i i}\left(x^{-1}\right) g_{j j}(y)=\operatorname{tr} \pi(x) \operatorname{tr} \pi(y) .
$$

Hence this integral is equal to

$$
\int_{G} \int_{G}|\overline{\operatorname{tr} \pi(x)} \operatorname{tr} \pi(y)|^{2} d x d y=\left(\int_{G}|\operatorname{tr} \pi(x)|^{2} d x\right)^{2}=1 / n^{2}
$$

since $\pi$ is irreducible.

It follows that $V$ acts irreducibly on each of the spaces spanned by $\left\{g_{i j}^{\mathbf{k}}: 1 \leqq i, j \leqq n_{k}\right\}$, for fixed $k$. Hence the previous theorem applies, and we can state our result, since these spaces are eigenspaces of $L$ by Schur's lemma.

Theorem 2. Let $G$ be a compact Lie group of dimension $n$, and let 
$s \geqq n / 4$ be an integer. If $f \in H_{2 s}$, and in particular if $f \in C^{2 s}(G)$, then the Fourier series for $f$ converges absolutely and uniformly.

NotE. Edward Nelson has pointed out that by repeated application of the reasoning used in the proof of Theorem 1, we can show that the Fourier series of a $C^{\infty}$ function on $G$ converges in the topology of $C^{\infty}$. It follows that the Fourier series of a distribution $u$ on $G$ converges weakly to $u$.

\section{BIBLIOGRAPHY}

1. Gong Shang, Fourier analysis on unitary groups. III, Chinese Math.-Acta 4 (1963), 169-178.

2. S. Helgason, Differential geometry and symmetric spaces, Academic Press, New York, 1962.

3. E. Nelson and W. F. Stinespring, Representations of elliptic operators in an enveloping algebra, Amer. J. Math. 81 (1959), 547-560.

Princeton University 